\title{
Two-Level Minimally Invasive Transforaminal Lumbar Interbody Fusion: Surgical Technique and Illustrative Cases
}

\author{
Rory J. Petteys, Jay Rhee, and Jean-Marc Voyadzis \\ Department of Neurosurgery, Georgetown University Hospital, 3800 Reservoir Road NW, PHC-7, Washington, DC 20007, USA \\ Correspondence should be addressed to Jean-Marc Voyadzis; jmvoyadzis@gmail.com
}

Received 9 July 2013; Accepted 28 July 2013

Academic Editors: Y. Izci, W. Kolb, and P. Spennato

Copyright (C) 2013 Rory J. Petteys et al. This is an open access article distributed under the Creative Commons Attribution License, which permits unrestricted use, distribution, and reproduction in any medium, provided the original work is properly cited.

\begin{abstract}
Transforaminal lumbar interbody fusion (TLIF) is a common procedure performed by spine surgeons. The indications for TLIF include back pain and radiculopathy as a consequence of canal or foraminal stenosis, degenerative disc disease, spondylolisthesis, or deformity. Minimally invasive techniques (MIS) have proven to be effective for single-level TLIF and are associated with less blood loss, fewer wound complications and infections, faster recovery, and decreased hospital cost. To date, there is very little data on 2-level MIS TLIF. We present our technique for 2-level MIS TLIF with case illustrations and a review of the literature.
\end{abstract}

\section{Introduction}

Lumbar arthrodesis is generally indicated in the treatment of spondylotic diseases of the spine including spondylolisthesis, degenerative disc disease, facet arthropathy, and scoliosis. While posterolateral fusion has been shown to be clinically efficacious and safe, many advocate interbody fusion because it affords the luxury of placing the graft under compression forces, can greatly restore lordosis and disc height, and permits indirect decompression and foraminal distraction [17].

Lumbar interbody fusion was first described by Cloward $[8,9]$ in 1952 via a posterior approach (PLIF), which proved effective in achieving interbody fusion but required significant nerve root and thecal sac retraction [10]. Harms and Rolinger subsequently described a less invasive approach in 1982 via transforaminal route (TLIF) [11]. This approach provides a more lateral point of access to the disc space, thus limiting retraction of the neural elements. While clinical outcomes have been favorable with both of these approaches, the amount of muscle dissection and retraction required to expose the spine was thought to adversely affect patient recovery [12-18]. As an alternative to these standard open techniques and with technological advancements in illumination, retractors, and magnification, Foley et al. introduced the minimally invasive (MIS) TLIF in an attempt to reduce muscle injury associated with the standard subperiosteal exposure $[1,2]$. This approach involved a paramedian incision and exploits natural anatomic corridors. As described, the MIS TLIF is an effective option for treatment of the aforementioned conditions that affect the lumbar spine.

Minimally invasive or minimal access techniques offer several advantages over their traditional open counterparts. The amount of muscle dissection and retraction necessary to expose the spine in a standard approach leads to significant muscle damage and atrophy [12-15, 17, 18]. MIS techniques do not require the same degree of muscle, fascia, and soft tissue disruption. Additionally, several authors report other advantages including less intraoperative blood loss, less postoperative pain and narcotic use, earlier ambulation, and decreased length of hospital stay [1-7]. The indications for 2-level MIS TLIF are similar and include multilevel degenerative disc disease, spondylolisthesis, and scoliosis. To date, the technique of 2-level MIS TLIF has not been described in the literature. What follows is the senior author's (J. M. Voyadzis) technique description of this approach with several case illustrations and perioperative clinical data.

\section{Surgical Technique}

The patient is brought to the operating room and undergoes general endotracheal anesthesia. A urethral catheter 
is inserted, pneumatic compression devices are applied to the lower legs, and neurophysiological monitoring leads are placed; an intraarterial line may be used for monitoring if indicated. The patient is then positioned prone on a Jackson flat top table with chest roll(s) to maintain lumbar lordosis. Preoperative antibiotics are administered. The fluoroscopic arm is then positioned such that the base of the device is on the side opposite to the surgeon. The surgeon should stand on the side of the interbody approach that is determined by the patient's symptoms.

Using anterior-posterior fluoroscopy, the midline is marked on the skin and a parallel line is drawn 4 to $4.5 \mathrm{~cm}$ lateral to the midline (Figures 1(a) and 1(b)). At the lower lumbar segments, this distance from the midline allows one to use the natural cleavage plane between the multifidus and longissimus muscles as advocated by Wiltse et al. [19]. Lateral fluoroscopy is then used to identify the correct levels. For 2-level TLIF, the center of the incision is planned at the midpoint between both target disc spaces to ensure a proper trajectory (Figure 1(c)). The length is the incision is dependent upon the size of the tubular retractor used. This is typically $2.5 \mathrm{~cm}$ with the Quadrant retractor (Medtronic, Memphis, TN, USA). The patient is then draped after antiseptic is applied and the skin is infiltrated with a local anesthetic.

2.1. Graft Insertion at the First Level. The level with the worst pathology or referable symptoms should be addressed first. The incision is made in the typical fashion. The underlying fascia is exposed and a longitudinal fasciotomy is performed with Bovie electrocautery to facilitate passage of dilators. The blunt end of a Kirschner wire or Steinmann pin is inserted along a slight medial trajectory under fluoroscopic guidance. The goal is to "dock" the wire on the ipsilateral facet joint of the level in question (Figure 1(d)). Serial dilators are then inserted over the wire followed by the tubular retractor, which in turn is secured to a table mounted arm. Final placement is then confirmed by fluoroscopy (Figures 1(e) and 1(f)).

Soft tissue within the working channel, which consists of adipose tissue and scant muscle, is removed with Bovie electrocautery. This exposes the lamina and facet complex. The lamino-facet junction should be in the lateral half of the working field and the lateral half of the lamina in the medial field. A sublaminar plane is defined with curettes and a laminotomy is performed followed by the removal of pars interarticularis and inferior facet (Figure $1(\mathrm{~g})$ ). This can be performed with the high-speed drill or with a series of osteotome cuts. This bone can be stripped of its adjoining soft tissue and morselized as autograft for fusion. The superior portion of the superior facet of the level below is removed in similar fashion and a working corridor to the disc space has now been defined. If a laminectomy for stenosis is necessary, the laminectomy is completed in standard fashion on the side of the approach. The working channel can then be "wanded" contralaterally and the table tilted. The inner aspect of the contralateral lamina is drilled and all associated ligamentum flavum is removed in order to achieve bilateral decompression.
The working channel is reoriented ipsilaterally and the table realigned. The disc space, traversing, and exiting nerve root and thecal sac are now in view and great care is taken to protect them. The thecal sac is gently retracted medially and all epidural veins coagulated so as to further expose the disc space. The disc space is then incised and a thorough discectomy is performed with curettes, rongeurs, and endplate shavers. The end plates are thoroughly prepared for fusion by removing the cartilaginous portion. Dilators are then employed to distract the disc space, increasing overall disc space height in preparation for the interbody grafting material (Figure 1(h)). The graft may consist of polyetherether ketone (PEEK), autograft, cadaver allograft, titanium or carbon-fiber cages, or absorbable materials. Graft placement can also be supplemented with recombinant human bone morphogenetic protein and morselized autograft.

2.2. Graft Insertion at the Second Level. After graft insertion at the first level is completed, the retractor is then oriented toward the other disc space and the exact same steps are repeated (Figure 1(i)). The location of the initial skin incision midway between the two discs is very important for 2-level approaches. If L5-S1 and L4-5 are being treated, the midpoint of the incision will typically be slightly more cephalad to have to a parallel trajectory to both disc spaces. If the incision is centered too close to one disc space, performing the second interbody graft placement will be made difficult due to the long reach required and difficult working angle. Another option is to extend the incision and insert a K-wire and dilators directed at the second level.

2.3. Pedicle Screw Insertion. The working channel is oriented back to the first interbody level. The junction of transverse process and facet joints of both pedicles is exposed through the retractor. Under direct visualization, a pilot hole is created with a high-speed drill at this junction with lateral imaging. A Jamshidi needle is then inserted into both pedicles followed by a $\mathrm{K}$-wire (Figures $1(\mathrm{j}), \mathrm{1}(\mathrm{k})$, and $1(\mathrm{l})$ ). The needles and working channel are subsequently removed.

Percutaneous pedicle screws are then inserted in standard fashion in all remaining pedicles using simultaneous AP and lateral imaging [20]. Briefly, an incision is marked using AP guidance approximately $2 \mathrm{~cm}$ lateral to the lateral border of the 4 remaining pedicles of interest. The underlying fascia is opened with Bovie electrocautery and a Jamshidi needle is positioned at the junction of the transverse process and facet joint. The needle is then tapped into the pedicle using simultaneous AP and lateral fluoroscopic imaging (Figure 1(l)). The inner stylet is removed and a $\mathrm{K}$-wire is inserted into the vertebral body (Figure $1(\mathrm{~m})$ ). A series of dilators are inserted over the K-wire followed by a cannulated tap and an appropriately sized cannulated pedicle screw (Figures $1(\mathrm{n})$ and $1(\mathrm{o})$ ). The screw heads are stimulated with electromyographic monitoring to evaluate for a pedicle breach. After proper placement is confirmed, an appropriately sized rod is inserted percutaneously and set screws are applied and tightened (Figure 1(p)). Compression can also be achieved here to restore lordosis prior to securing 


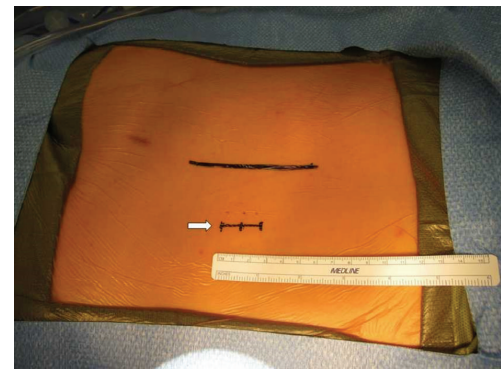

(a)

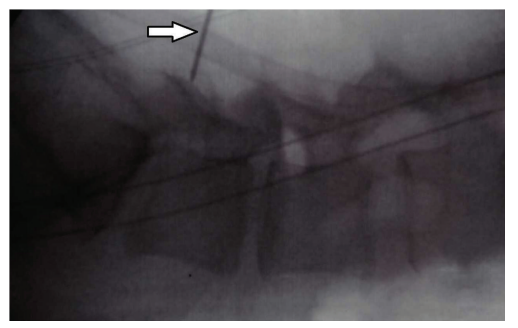

(c)

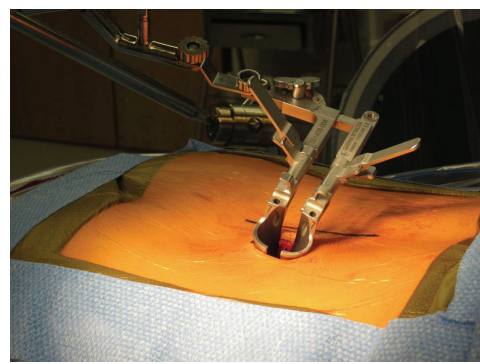

(e)

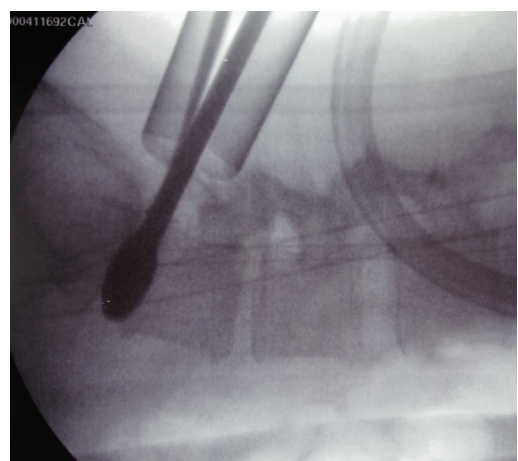

(g)

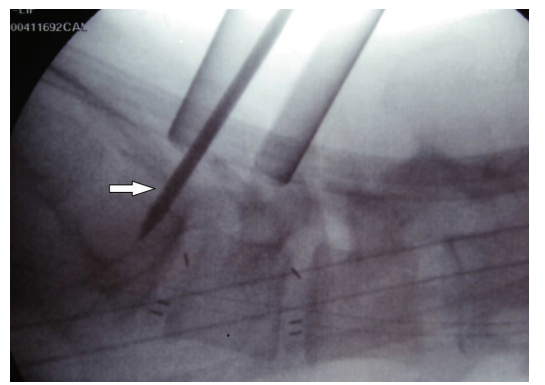

(i)

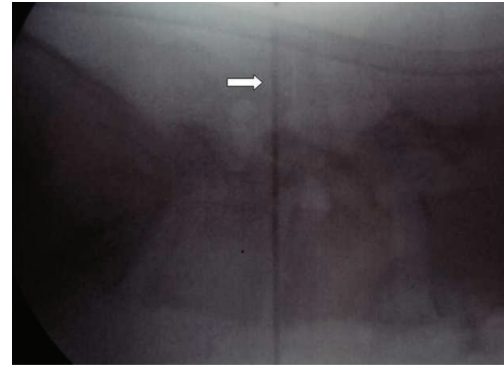

(b)

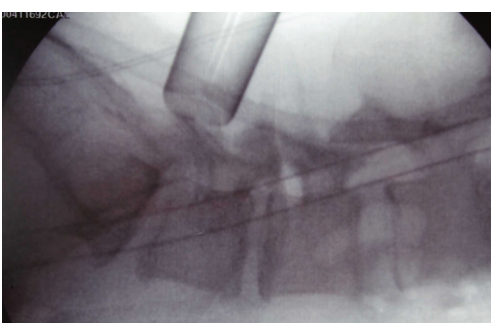

(d)

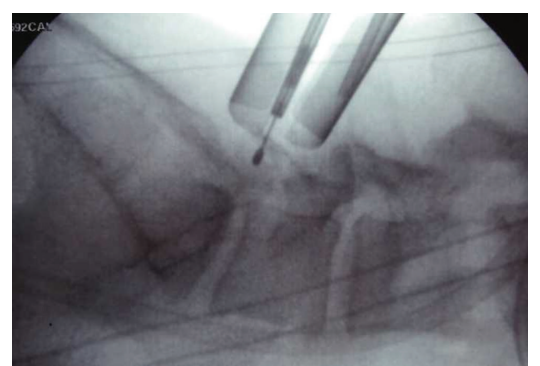

(f)

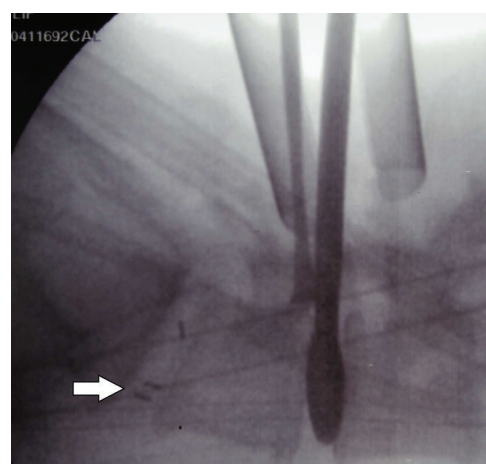

(h)

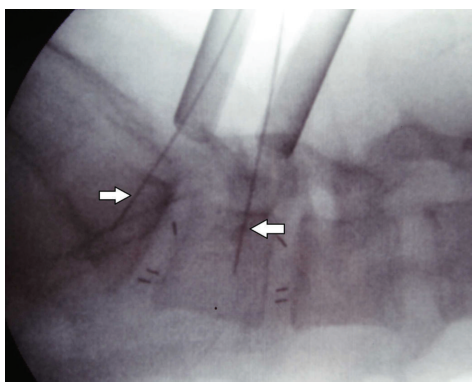

(j)

FIgure 1: Continued. 


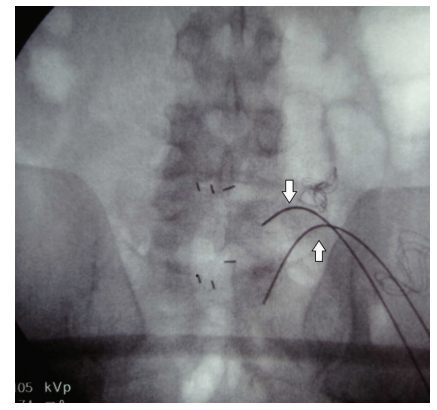

(k)

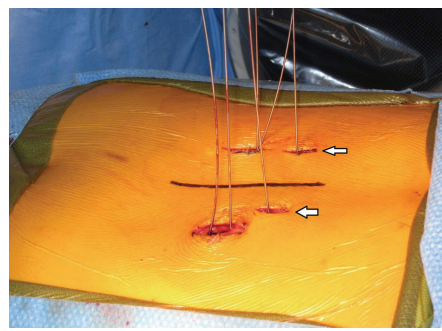

$(\mathrm{m})$

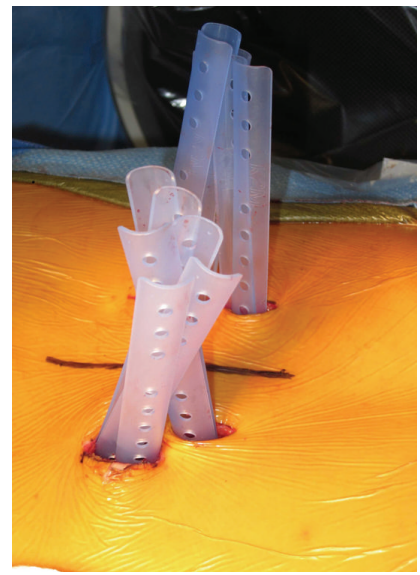

(o)

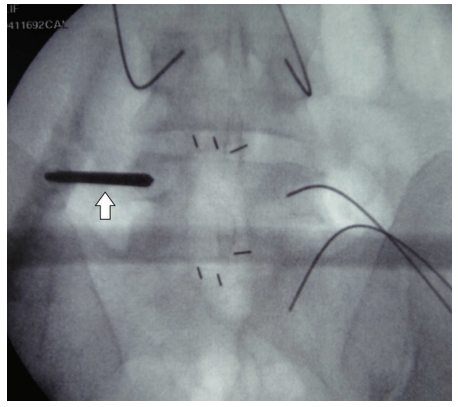

(1)

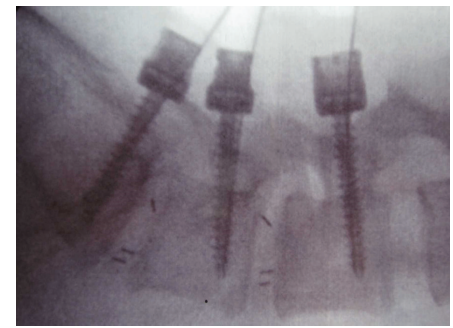

(n)

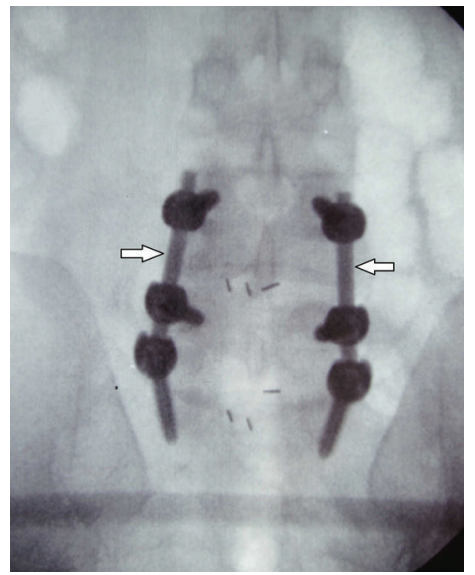

(p)

Figure 1: (a) A $2.5 \mathrm{~cm}$ incision is drawn approximately $4.5 \mathrm{~cm}$ away from the midline. This approach finds a natural cleavage plane in the paraspinal musculature advocated by Wiltse. (b) An intraoperative lateral fluoroscopic image of a patient in the prone position. For a 2level MIS TLIF at L4-5 and L5-S1, the center of the skin incision usually lies closer to the L4-5 disc space to gain proper access to both levels. A K-wire (arrow) is used to plan the skin incision. (c) Lateral view showing the K-wire (arrow) docked on the L5-S1 facet complex. (d) Lateral view after tubular dilator insertion and retractor placement at L5-S1. (e) Intraoperative photograph of the final position of the tubular retractor secured by a table mounted arm. (f) Lateral view showing the removal of the L5-S1 facet complex with a high speed drill through the tubular retractor. $(\mathrm{g})$ Lateral view after insertion of a trial into the disc space for sizing of the spacer and disc dilation. (h) Lateral view after L5-S1 interbody graft placement (arrow) and trial insertion into the L4-5 disc space. (i) Lateral view after 2-level graft placement. The retractor is oriented back towards L5-S1 for transpedicular Jamshidi needle (arrow) insertion at S1. ((j) and (k)) Lateral (j) and AP (k) views revealing the insertion of $\mathrm{K}$-wires (arrows) through the retractor in preparation for percutaneous pedicle screw placement. (l) AP view showing multilevel K-wire insertion and percutaneous transpedicular Jamshidi needle placement. Note the insertion point of the needle at the junction of the transverse process and the facet joint at L5. (m) An intraoperative photograph after all K-wires are placed. (n) Lateral view showing percutaneous pedicle screw placement on one side. (o) Intraoperative photograph after placement of all pedicle screws from L4-S1. The plastic screw extenders aid in the guidance of rod placement. Once the rods are placed percutaneously and secured with sets crews, the plastic retractors are removed. (p) Final AP view. 


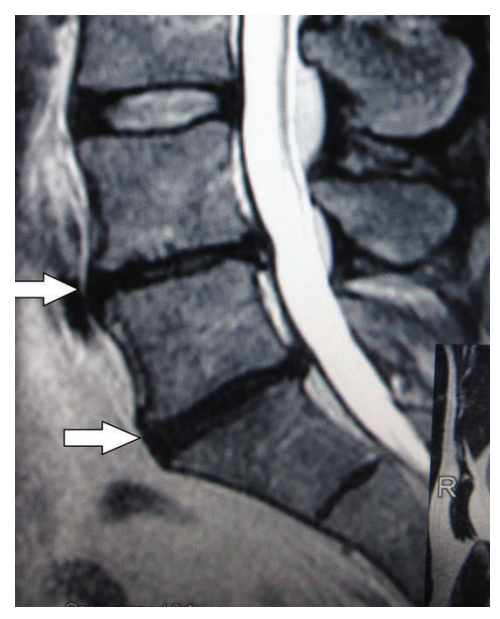

(a)

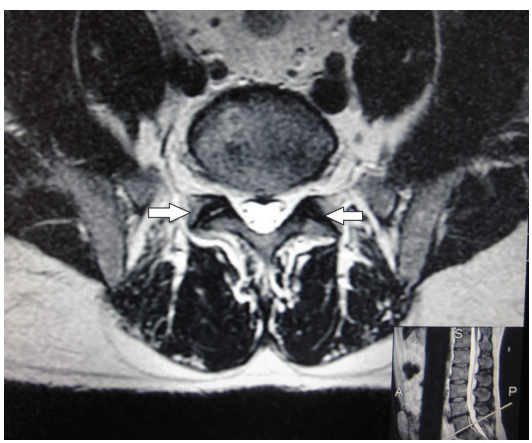

(c)

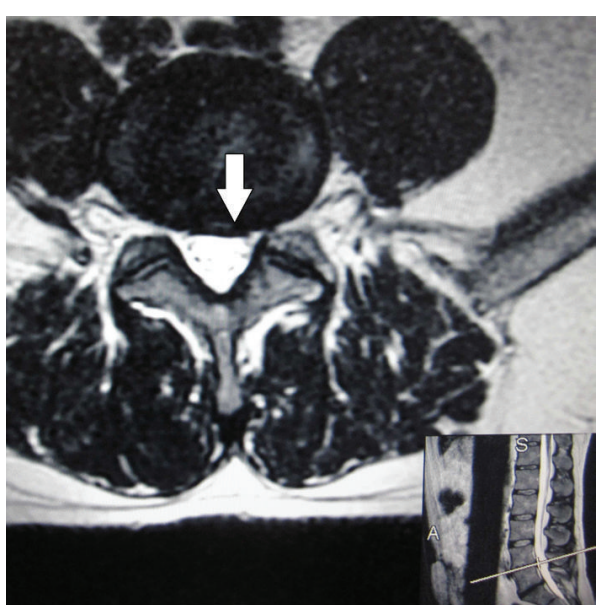

(b)

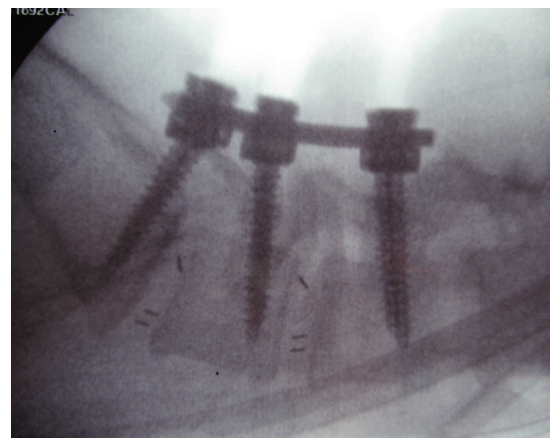

(d)

Figure 2: ((a), (b), and (c)) Sagittal (a) and axial ((b) and (c)) T2 weighted MRI revealing significant degenerative disc disease (arrows) at L4-5 and L5-S1 without stenosis. (d) Lateral view after placement of the interbody grafts, percutaneous pedicle screws, and rods.

the rod. The lumbo-dorsal fascia and skin are then closed in layers.

\section{Case Illustrations}

3.1. Case 1. A 38-year-old healthy man presented with a 15-year history of increasingly severe low back pain. He noted occasional radiating left leg pain and mild associated numbness. These symptoms had a significant impact on his activities of daily living. He underwent extensive conservative management including physical therapy, numerous epidural injections, nonsteroidal anti-inflammatory medications, and narcotics which had provided limited relief. His physical examination demonstrated no neurologic deficits. A discogram showed concordant pain elicited at both L4-5 and L5-S1 with a negative control at L3-4. MRI studies revealed significant degenerative disc disease at L4-5 and L5-S1 with loss of disc height and associated endplate changes most pronounced at L4-5 (Figures 2(a), 2(b), and 2(c)). There was also a paracentral disc herniation at L4-5 (Figure 2(b)). He underwent a minimally invasive L4-5 and L5-S1 transforaminal lumbar interbody fusion with percutaneous pedicle screw instrumentation (Figure 2(d)). The operative time, estimated blood loss, and length of hospital stay were 4 hours 35 minutes, $200 \mathrm{~mL}$, and 48 hours, respectively. At his one-year follow-up visit, he reported complete resolution of both his back and leg pain.

3.2. Case 2. A 42-year-old woman presented with severe low back pain radiating to the lower extremities bilaterally worsening over five years. She noted that the pain began in her lower back and radiated to the posterolateral aspects of the thigh and calf bilaterally with the left side being more severe. Her pain was exacerbated with prolonged standing or walking as well as coughing. She described leg paresthesias in the same distribution of her pain. She also noted a general feeling of weakness in both of her legs. She had undergone physical therapy and epidural injections which provided limited benefit. She had taken narcotics and muscle relaxers to manage her pain. MRI studies of her lumbar spine revealed grade-1 L4-5 spondylolisthesis with severe lateral recess and foraminal stenosis (Figure 3(a)). There was also marked facet arthropathy and facet effusions (Figure 3(b)). At L5-S1, there was significant degenerative disc disease with a left paracentral disc protrusion (Figure 3(c)). She underwent a minimally invasive L4-5 and L5-S1 transforaminal lumbar interbody fusion with percutaneous pedicle screw instrumentation and an L4-5 decompressive laminectomy (Figures 3(f), 3(g), and 3(h)). The operative time, estimated 


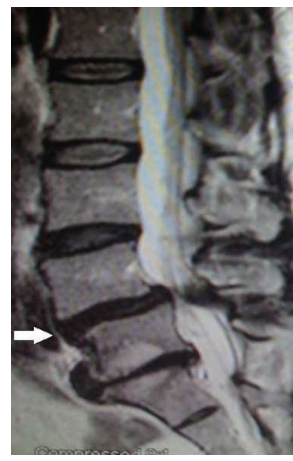

(a)

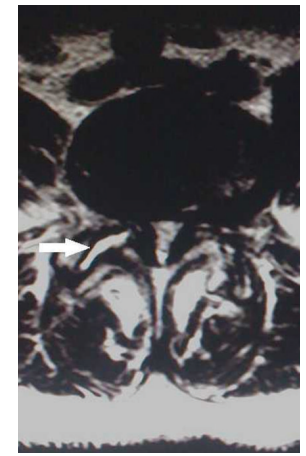

(b)

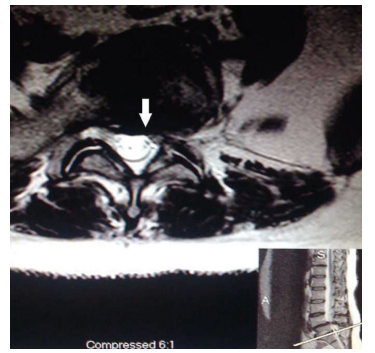

(c)

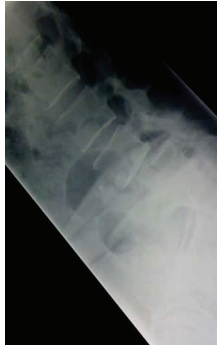

(d)

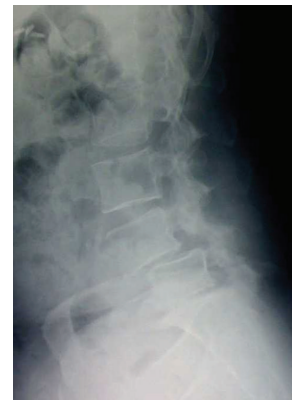

(e)

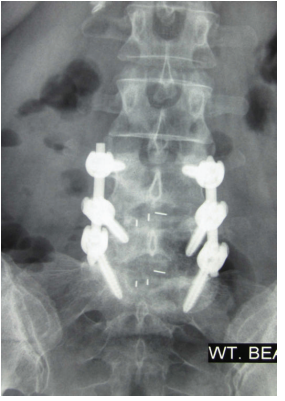

(f)

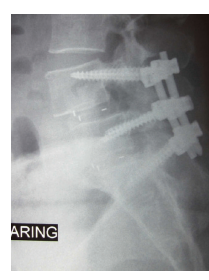

(g)

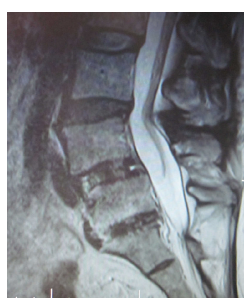

(i)

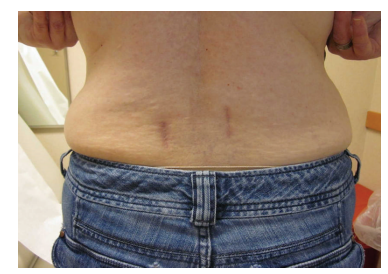

(h)

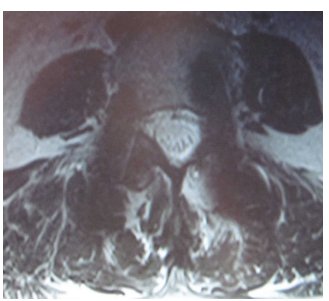

(j)

FIGURE 3: ((a), (b), and (c)) Sagittal (a) and axial ((b) and (c)) T2 weighted MRI imaging revealing degenerative disc disease at L4-5 and L5-S1 as well as a grade-1 spondylolisthesis at L4-5 (arrow) with multilevel stenosis. ((d) and (e)) Dynamic lateral X-rays revealing a grade-1 L4-5 spondylolisthesis that increases with flexion. (f) and (g) Postoperative AP and lateral X-rays after 2-level MIS TLIF. (h) Photograph at one year followup revealing well-healed incisions. ((i) and (j)) Sagittal ((i) and axial (j)) T2 weighted MRI revealing resolution of her multilevel stenosis and minimal postoperative atrophy and disruption of the paraspinal musculature $(\mathrm{j})$. 
blood loss, and length of stay were 4 hours 52 minutes, $300 \mathrm{~mL}$, and 72 hours, respectively. She enjoyed complete resolution of her back and bilateral lower extremity pain and paresthesias at her one-year follow-up visit. A postoperative MRI revealed resolution of her stenosis and minimal disruption of the paraspinal musculature (Figures 3(i) and 3(j)).

\section{Discussion}

Low back pain is a common complaint among adults all over the world and many of these patients require surgical treatment. Lumbar fusion, via TLIF or other techniques, is utilized to treat a variety of conditions including degenerative disc disease, facet arthropathy, spondylolisthesis, scoliosis, tumors, fractures, and others. The TLIF approach is a wellestablished method for achieving circumferential arthrodesis through a single, posterior approach, and recent advancements have allowed the procedure to be performed with minimally invasive techniques. Initial cadaveric studies of the MIS TLIF were first performed by Khoo and colleagues in 2002, demonstrating the safety and effectiveness of the procedure [5]. A follow-up study in 2005 showed significant decreases in intraoperative blood loss, postoperative narcotic use, and length of hospital stay in patients undergoing MIS TLIF compared to open lumbar fusion [4], thus establishing the validity of the technique.

Several studies since then have also demonstrated benefits of MIS TLIF over standard open techniques. Scheufler et al. demonstrated similar operative times and fusion rates but statistically significant reductions in blood loss and postoperative pain in patients who had MIS TLIF procedures [21]. There were no differences in clinical outcomes at 8 and 16 months as measured by standard questionnaires. Several other studies have also reported decreased intraoperative blood loss, postoperative narcotic use, and hospital stay in MIS TLIF patients, while achieving similar fusion rates and clinical outcomes as open procedures [22, 23]. Ghahreman et al. also reported earlier independent ambulation in patients who had MIS TLIF than patients who had open fusion [22]. Several recent reports by McGirt and colleagues have demonstrated the cost effectiveness of MIS fusion when compared to open procedures [24-26].

Minimally invasive techniques (including MIS TLIF) may be particularly advantageous in obese patients. These patients are particularly prone to wound and surgical site complications in open lumbar surgery because of the larger incision required and larger cavity created to expose the deep spine [27-30]. By using a tubular retractor, the spine may be accessed through the same size incision in an obese patient as in a nonobese patient. Obese patients also experience other benefits afforded by minimally invasive surgery-decreased blood loss and narcotic use-and one study found that BMI had no significant relationship with self-reported outcome measures, operative time, hospital stay, and complications [31]. Obese patients do present some challenges including poor fluoroscopic visualization of anatomical landmarks and limitations of the length of tubular retractors.
While the potential benefits reported on single-level MIS TLIF can be extrapolated to 2-level surgeries so can the potential drawbacks. A TLIF achieved through a narrow tubular retractor is one of the more technically challenging MIS surgeries. During the learning curve, one can expect greater operating times and a higher rate of complications such as cerebrospinal fluid leak [32]. Hardware related complications are also more common during this period [33]. Performing an additional complete facetectomy, disc preparation, and interbody graft placement after redirecting the retractor adds to this learning curve and technical difficulty. In addition, multilevel percutaneous instrumentation requires patience a greater degree of fluoroscopic precision and can also contribute to an increase in operative time and radiation exposure. In the illustrative cases, the mean operative time, estimated blood loss, and length of hospital stay were 4 hours 43 minutes, $250 \mathrm{~mL}$, and 2 days 12 hours, respectively. No transfusions were required and their length of stay was short. Both patients enjoyed complete resolution of their preoperative back and leg symptoms at one-year follow-up.

There are circumstances where 2-level MIS TLIF may not be indicated. Three-level disease may be best treated in the standard fashion as the additional operative time and radiation exposure may not outweigh the benefits. Patients with severe osteoporosis, high-grade spondylolisthesis, and severe scoliosis, where localization under fluoroscopy can be difficult due to anatomical irregularities, may be considered a contraindication. Additionally, very thin patients with little subcutaneous fat may not benefit from MIS procedures as the amount of muscle dissection required may actually be greater than standard open subperiosteal exposure.

\section{Conclusion}

We describe the technique of 2-level MIS TLIF via a single incision followed by bilateral percutaneous pedicle screw insertion. This report illustrates the feasibility and safety of the 2-level MIS TLIF technique. Data from single level MIS TLIF showing a reduction in morbidity and hospital stays can be extrapolated to 2-level MIS TLIFs. Additional studies with larger samples and followup are needed to further assess the efficacy of this method.

\section{References}

[1] K. T. Foley, L. T. Holly, and J. D. Schwender, "Minimally invasive lumbar fusion," Spine, vol. 28, pp. S26-S35, 2003.

[2] K. T. Foley and M. A. Lefkowitz, "Advances in minimally invasive spine surgery," Clinical Neurosurgery, vol. 49, pp. 499-517, 2002.

[3] J. W. German and K. T. Foley, "Minimal access surgical techniques in the management of the painful lumbar motion segment," Spine, vol. 30, no. 16, pp. S52-S59, 2005.

[4] R. E. Isaacs, V. K. Podichetty, P. Santiago et al., "Minimally invasive microendoscopy-assisted transforaminal lumbar interbody fusion with instrumentation," Journal of Neurosurgery, vol. 3, no. 2, pp. 98-105, 2005. 
[5] L. T. Khoo, S. Palmer, D. T. Laich, and R. G. Fessler, "Minimally invasive percutaneous posterior lumbar interbody fusion," $\mathrm{Neu}$ rosurgery, vol. 51, no. 5, pp. 166-181, 2002.

[6] K.-T. Kim, S.-H. Lee, K.-S. Suk, and S.-C. Bae, “The quantitative analysis of tissue injury markers after mini-open lumbar fusion," Spine, vol. 31, no. 6, pp. 712-716, 2006.

[7] J. D. Schwender, L. T. Holly, D. P. Rouben, and K. T. Foley, "Minimally invasive transforaminal lumbar interbody fusion (TLIF): technical feasibility and initial results," Journal of Spinal Disorders and Techniques, vol. 18, no. 1, pp. S1-S6, 2005.

[8] R. B. Cloward, "The treatment of ruptured lumbar intervertebral disc by vertebral body fusion. III. Method of use of banked bone," Annals of Surgery, vol. 136, no. 6, pp. 987-992, 1952.

[9] R. B. Cloward, "The treatment of ruptured lumbar intervertebral discs by vertebral body fusion. I. Indications, operative technique, after care," Journal of Neurosurgery, vol. 10, no. 2, pp. 154-168, 1953.

[10] C. D. Ray, “Threaded titanium cages for lumbar interbody fusions," Spine, vol. 22, no. 6, pp. 667-680, 1997.

[11] J. Harms and H. Rolinger, "A one-stage procedure in operative treatment of spondylolisthesis: dorsal traction-reposition and anterior fusion," Zeitschrift fur Orthopadie und Ihre Grenzgebiete, vol. 120, no. 3, pp. 343-347, 1982.

[12] G. Datta, K. K. Gnanalingham, D. Peterson et al., "Back pain and disability after lumbar laminectomy: is there a relationship to muscle retraction?" Neurosurgery, vol. 54, no. 6, pp. 1413-1420, 2004.

[13] R. Gejo, H. Matsui, Y. Kawaguchi, H. Ishihara, and H. Tsuji, "Serial changes in trunk muscle performance after posterior lumbar surgery," Spine, vol. 24, no. 10, pp. 1023-1028, 1999.

[14] Y. Kawaguchi, H. Matsui, and H. Tsuji, "Back muscle injury after posterior lumbar spine surgery: part 1: histologic and histochemical analyses in rats," Spine, vol. 19, no. 22, pp. 25902597, 1994.

[15] Y. Kawaguchi, H. Matsui, and H. Tsuji, "Back muscle injury after posterior lumbar spine surgery: part 2: histologic and histochemical analyses in humans," Spine, vol. 19, no. 22, pp. 2598-2602, 1994.

[16] T. G. Mayer, H. Vanharanta, R. J. Gatchel et al., "Comparison of CT scan muscle measurements and isokinetic trunk strength in postoperative patients," Spine, vol. 14, no. 1, pp. 33-36, 1989.

[17] T. Sihvonen, A. Herno, L. Paljarvi, O. Airaksinen, J. Partanen, and A. Tapaninaho, "Local denervation atrophy of paraspinal muscles in postoperative failed back syndrome," Spine, vol. 18, no. 5, pp. 575-581, 1993.

[18] J. R. Styf and J. Willén, "The effects of external compression by three different retractors on pressure in the erector spine muscles during and after posterior lumbar spine surgery in humans," Spine, vol. 23, no. 3, pp. 354-358, 1998.

[19] L. L. Wiltse, J. G. Bateman, R. H. Hutchinson, and W. E. Nelson, "The paraspinal sacrospinalis-splitting approach to the lumbar spine," Journal of Bone and Joint Surgery A, vol. 50, no. 5, pp. 919-926, 1968.

[20] K. T. Foley and S. K. Gupta, "Percutaneous pedicle screw fixation of the lumbar spine: preliminary clinical results," Journal of Neurosurgery, vol. 97, no. 1, pp. 7-12, 2002.

[21] K.-M. Scheufler, H. Dohmen, and V. I. Vougioukas, "Percutaneous transforaminal lumbar interbody fusion for the treatment of degenerative lumbar instability," Neurosurgery, vol. 60, no. 4, pp. S-203-S-212, 2007.
[22] A. Ghahreman, R. D. Ferch, P. J. Rao, and N. Bogduk, "Minimal access versus open posterior lumbar interbody fusion in the treatment of spondylolisthesis," Neurosurgery, vol. 66, no. 2, pp. 296-304, 2010.

[23] C. W. B. Peng, W. M. Yue, S. Y. Poh, W. Yeo, and S. B. Tan, "Clinical and radiological outcomes of minimally invasive versus open transforaminal lumbar interbody fusion," Spine, vol. 34, no. 13, pp. 1385-1389, 2009.

[24] O. Adogwa, S. L. Parker, A. Bydon, J. Cheng, and M. J. McGirt, "Comparative effectiveness of minimally invasive versus open transforaminal lumbar interbody fusion: 2-year assessment of narcotic use, return to work, disability, and quality of life," Journal of Spinal Disorders and Techniques, vol. 24, no. 8, pp. 479-484, 2011.

[25] S. L. Parker, O. Adogwa, A. Bydon, J. Cheng, and M. J. McGirt, "Cost-effectiveness of minimally invasive versus open transforaminal lumbar interbody fusion for degenerative spondylolisthesis associated low-back and leg pain over two years," World Neurosurgery, vol. 78, pp. 178-184, 2012.

[26] S. L. Parker, S. K. Mendenhall, D. N. Shau et al., "Minimally invasive versus open transforaminal lumbar interbody fusion (TLIF) for degenerative spondylolisthesis: comparative effectiveness and cost-utility analysis," World Neurosurgery, 2013.

[27] M. A. Olsen, J. Mayfield, C. Lauryssen et al., "Risk factors for surgical site infection in spinal surgery," Journal of Neurosurgery, vol. 98, no. 2, pp. 149-155, 2003.

[28] N. Patel, B. Bagan, S. Vadera et al., "Obesity and spine surgery: relation to perioperative complications," Journal of Neurosurgery, vol. 6, no. 4, pp. 291-297, 2007.

[29] A. E. Telfeian, G. T. Reiter, S. R. Durham, and P. Marcotte, "Spine surgery in morbidly obese patients," Journal of Neurosurgery, vol. 97, no. 1, pp. 20-24, 2002.

[30] C. Wimmer, H. Gluch, M. Franzreb, and M. Ogon, "Predisposing factors for infection in spine surgery: a survey of 850 spinal procedures," Journal of Spinal Disorders, vol. 11, no. 2, pp. 124128, 1998.

[31] D. S. Rosen, S. D. Ferguson, A. T. Ogden, D. Huo, and R. G. Fessler, "Obesity and self-reported outcome after minimally invasive lumbar spinal fusion surgery," Neurosurgery, vol. 63, no. 5, pp. 956-960, 2008.

[32] J.-M. Voyadzis, "The learning curve in minimally invasive spinal surgery," Seminars in Spine Surgery, vol. 23, no. 1, pp. 9-13, 2011.

[33] A. Habib, Z. A. Smith, C. D. Lawton, and R. G. Fessler, "Minimally invasive transforaminal lumbar interbody fusion: a perspective on current evidence and clinical knowledge," Minimally Invasive Surgery, vol. 2012, Article ID 657342, 9 pages, 2012. 


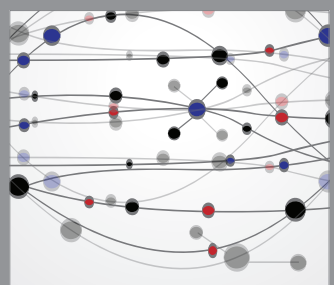

The Scientific World Journal
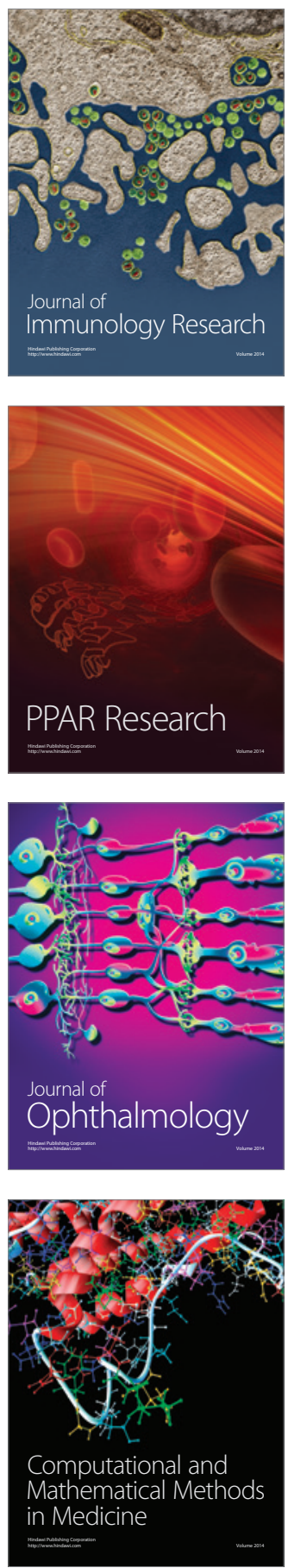

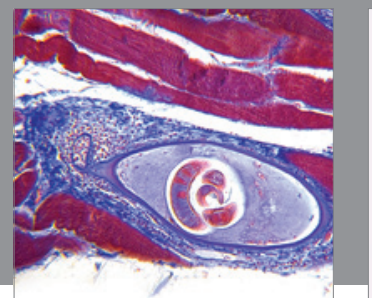

Gastroenterology

Research and Practice
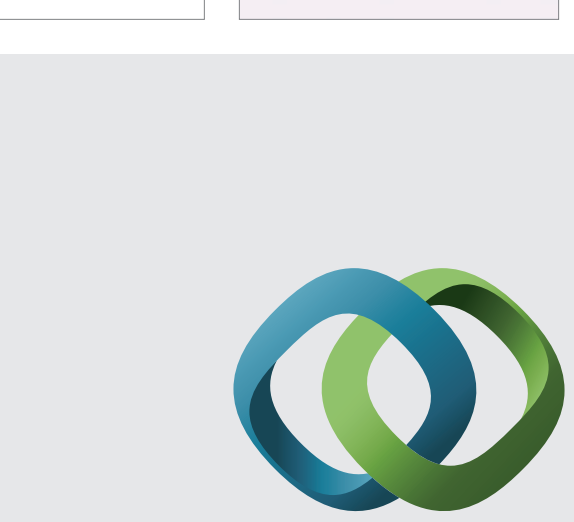

\section{Hindawi}

Submit your manuscripts at

http://www.hindawi.com
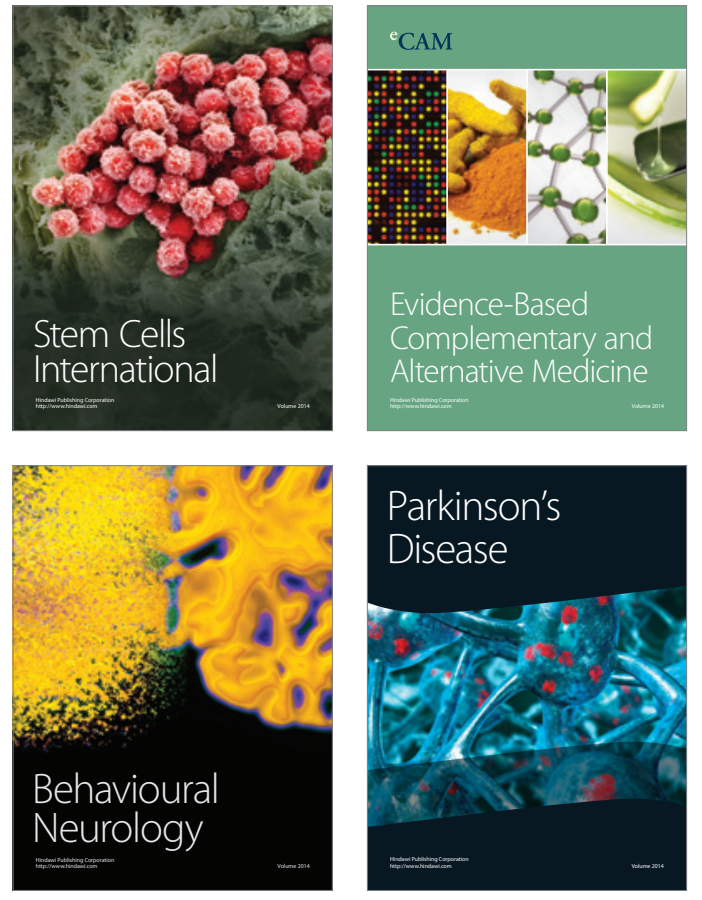
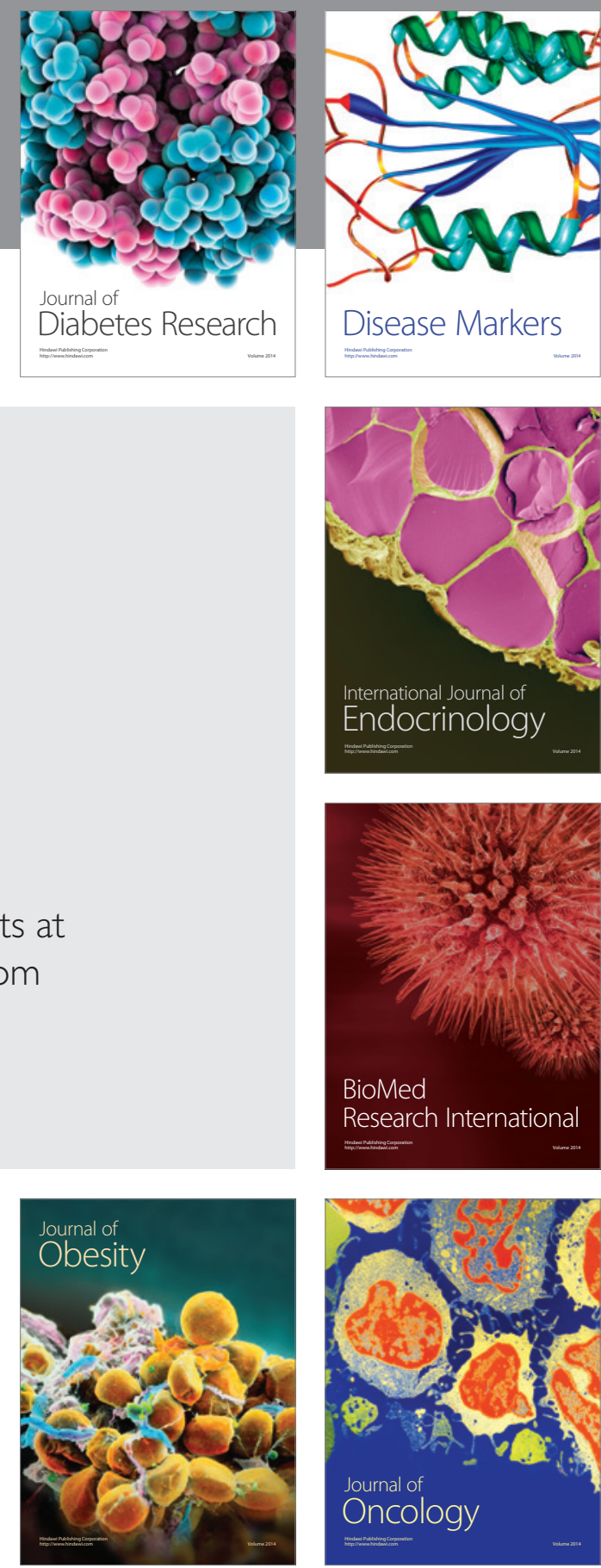

Disease Markers
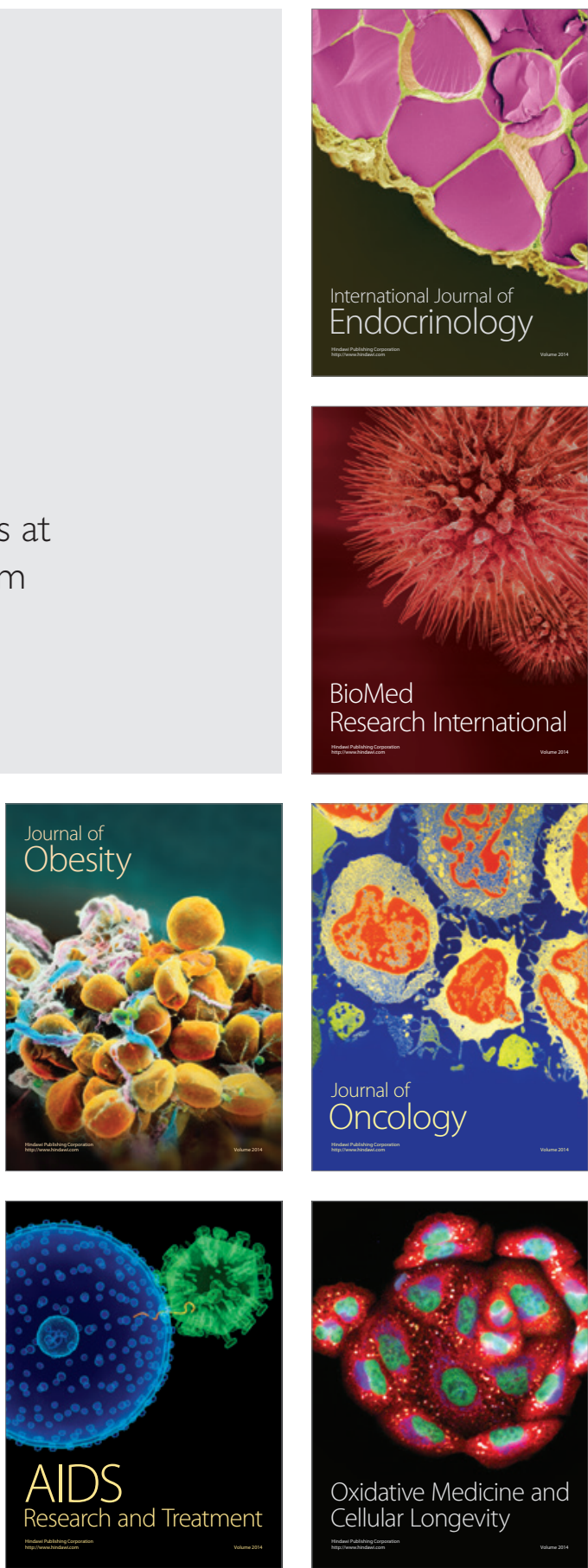